\title{
EDITORIAL
}

Check for updates

\section{Break the taboo with poo}

\author{
We need to talk about poo. Everybody does it, and research into defaecation and associated \\ disorders deserves more attention.
}

Whether you squat, sit or empty into a pouch, everybody needs to defaecate to remove faecal waste from their body. And yet this physiological process remains a taboo. If poo and defaecation were more widely discussed, then the stigma that can be associated with this process and the related gastrointestinal conditions ${ }^{1}$ could be lessened. What do we know about how we poo?

In their Review, Heitmann et al. ${ }^{2}$ describe the physiology of human defaecation and its role in disorders of continence and evacuation. Defaecation is a complex and coordinated process that integrates multiple physiological systems, including neuromuscular, hormonal and cognitive systems ${ }^{2}$. Consisting of four phases (basal, pre-expulsive, expulsive and end), the underlying processes can start up to an hour before the actual point of expulsion. There is a wide range when it comes to the frequency of defaecation and for healthy adults it is most commonly reported as the ' 3 and 3 ' metric of normal frequency - from three bowel movements per day to three bowel movements per week ${ }^{3}$. Given the complexity of this multisystem process it is not surprising that disorders of defaecation are common. According to a Rome Foundation survey that included 73,076 adults from 33 countries across 6 continents, $40.3 \%$ of people worldwide reported a disorder of gut-brain interaction (previously known as functional gastrointestinal disorder), which includes several bowel disorders that influence defaecation ${ }^{4}$. In the same survey, depending on what criteria was used for the definition or subtype, the pooled prevalence of irritable bowel syndrome (which can include symptoms of constipation, bloating, pain and diarrhoea) was $0.3-4.1 \%$ and $11.7 \%$ for functional constipation ${ }^{4}$.

However, not all that we excrete is necessarily 'waste'. Human excreta (including urine and faeces) contain a rich source of biomarkers of health that potentially could be harnessed for disease prevention or early detection ${ }^{5}$. Blood in stool can be an indicator of colorectal cancer ${ }^{6}$ and many bowel cancer screening programmes already incorporate faecal immunochemical tests as part of home testing, which can improve participation in screening? Development of 'smart' toilets for personalized health monitoring is already underway ${ }^{8,9}$. Furthermore, the growing understanding of the role of the gut microbiome in human health and disease, most often analysed via faecal samples, has led to interest in developing the gut microbiome as a means of individualizing treatment strategies, including roles in diagnosis and as a source of novel therapies itself ${ }^{10}$. The song 'Everything comes down to poo', which featured in an episode of the TV show Scrubs and had the refrain "check the poo", was remarkably prescient in that regard.

In the UK, the COVID-19 pandemic, national lockdowns and temporary closing of hospitality and public toilet facilities highlighted the precarity of access to toilets for the public - an issue well known to those with bladder and bowel conditions when sometimes you 'just can't wait' - and led to the development of a crowd-sourced website as a means to find a 'lockdown loo', but now enables people to search for open toilet facilities across the region. However, as it stands, billions worldwide still lack access to safely managed sanitation, with poor sanitation linked to infectious disease transmission as well as reduced well-being and social and economic development ${ }^{11}$. Each year, 19 November marks World Toilet Day, an observance set up by the United Nations to celebrate toilets, to raise awareness and to take action for the global sanitation crisis; the ultimate aim is achieving Sustainable Development Goal 6, to "ensure access to water and sanitation for all", by 2030. Whatever your 'throne' may be, more open discussion of defaecation and issues associated with gastrointestinal diseases is needed, as well as research into this fundamental human process.

1. Hearn, M., Whorwell, P. J. \& Vasant, D. H. Stigma and irritable bowel syndrome: a taboo subject? Lancet Gastroenterol. Hepatol. 5, 607-615 (2020).

2. Heitmann, P. T. et al. Understanding the physiology of human defaecation and disorders of continence and evacuation. Nat. Rev. Gastroenterol. Hepatol. https://doi.org/10.1038/s41575-02100487-5 (2021)

3. Mitsuhashi, S. et al. Characterizing normal bowel frequency and consistency in a representative sample of adults in the United States (NHANES). Am. J. Gastroenterol. 113, 115-123 (2018).

4. Sperber, A. D. et al. Worldwide prevalence and burden of functional gastrointestinal disorders, results of Rome Foundation Global Study. Gastroenterology 160, 99-114 (2021).

5. Park, S.-M. Digital biomarkers in human excreta. Nat. Rev. Gastroenterol. Hepatol 18, 521-522 (2021).

6. Kuipers, E. J. et al. Colorectal cancer. Nat. Rev. Dis. Primers 1, 15065 (2015).

7. Quintero, E. et al. Colonoscopy versus fecal immunochemical testing in colorectal-cancer screening. N. Engl. J. Med. 366, 697-706 (2012).

8. Wang, X. J. \& Camilleri, M. A smart toilet for personalized health monitoring. Nat. Rev. Gastroenterol. Hepatol. 17, 453-454 (2020)

9. Park, S.-M. et al. A mountable toilet system for personalized health monitoring via the analysis of excreta. Nat. Biomed. Eng. 4, 624-635 (2020).

10. Schupack, D. A. et al. The promise of the gut microbiome as part of individualized treatment strategies. Nat. Rev. Gastroenterol. Hepatol. https://doi.org/10.1038/s41575-021-00499-1 (2021).

11. World Health Organization. Sanitation. World Health Organization https://www.who.int/news-room/fact-sheets/detail/sanitation (2019). 